\title{
MANIPULATIONS, HARCÈLEMENTS ET COMPLOTS : UNE PARANOÏSATION IMAGINAIRE DU LIEN SOCIAL CONTEMPORAIN ?
} Thierry Lamote, Romuald Hamon

Groupe d'études de psychologie | « Bulletin de psychologie »

2016/5 Numéro 545 | pages 381 à 396

ISSN 0007-4403

Article disponible en ligne à l'adresse :

http://www.cairn.info/revue-bulletin-de-psychologie-2016-5-page-381.htm

\section{Pour citer cet article :}

Thierry Lamote, Romuald Hamon, « Manipulations, harcèlements et complots : une paranoïsation imaginaire du lien social contemporain ? », Bulletin de psychologie 2016/5 (Numéro 545), p. 381-396.

DOI 10.3917/bupsy.545.0381

Distribution électronique Cairn.info pour Groupe d'études de psychologie.

(C) Groupe d'études de psychologie. Tous droits réservés pour tous pays.

La reproduction ou représentation de cet article, notamment par photocopie, n'est autorisée que dans les limites des conditions générales d'utilisation du site ou, le cas échéant, des conditions générales de la licence souscrite par votre établissement. Toute autre reproduction ou représentation, en tout ou partie, sous quelque forme et de quelque manière que ce soit, est interdite sauf accord préalable et écrit de l'éditeur, en dehors des cas prévus par la législation en vigueur en France. Il est précisé que son stockage dans une base de données est également interdit. 


\title{
Manipulations, harcèlements et complots : une paranoïsation imaginaire du lien social contemporain ?
}

\author{
LAMOTE Thierry a \\ HAMON Romuald ${ }^{b}$
}

${ }^{a}$ Centre de Recherches Psychanalyse, médecine et société, EA 3522, Université Paris Diderot-Paris 7 , France.

${ }^{\mathrm{b}}$ Laboratoire de psychopathologie et clinique psychanalytique, EA 4050, Recherches en psychopathologie : nouveaux symptômes et lien social, Université Rennes 2, France.

Correspondance : Thierry Lamote, 19 avenue des Pyrénées, 64100 Bayonne, France.

Courriel : thierry_lamote@yahoo.fr

Texte reçu le 9 septembre 2015 et accepté le 21 décembre 2015
Résumé : Ce travail se propose d'interroger le schéma d'analyse des phénomènes sectaires fondé sur la notion de manipulation mentale. Cette notion s'est en effet imposée, ces dernières années, dans le discours médico-légal et dans le champ des sciences humaines et sociales, au point de se voir progressivement appliquer à des phénomènes aussi distincts que les sectes, la radicalisation islamiste, jusqu'aux malaises professionnels et domestiques, via les théories du harcèlement moral. Après avoir dévoilé ce qui forme le noyau de ce schéma d'analyse, à savoir un fantasme de structure perverse, nous tenterons de discerner quelle part a eue, dans cet abord des phénomènes sociaux, l'invention de la catégorie de "perversion narcissique ». Nous analyserons alors ce que le surgissement, dans la série des doctrines usant du schème manipulatoire, de trois figures actuelles du Père jouisseur (le pervers narcissique, le gourou manipulateur, le harceleur), enseigne concernant la paranoïsation du lien social contemporain. Nous conclurons en interrogeant l'incidence de cette figure et de ces doctrines sur les phénomènes de radicalisation.

Manipulation, Harassment and Plots: An Imaginary Paranoization of Contemporary Social Bonds?

Abstract: This study seeks to question the analytical framework applied to cults based on the notion of mental manipulation. This notion has become pervasive in recent years in the forensic discourse, as well as in the fields of the humanities and the social sciences, to such an extent that it has gradually been applied to phenomena as diverse as cults, Islamic radicalization, and even professional and domestic conflicts, through the theories of psychological harassment. After having exposed what stands at the heart of this analytical framework - a fantasy of a perverse structure-we will attempt to assess what part the invention of the category of 'narcissistic perversion' has had in this approach to social phenomena. We will then analyze what can be learned about the paranoization of contemporary social bonds from the emergence of three prevailing figures of the Père jouisseur (the narcissistic pervert, the manipulative guru, and the harasser) in doctrines employing the framework of manipulation. We will conclude this study by discussing the effects of these figures and doctrines on the phenomenon of radicalization. 
Peu après les attentats terroristes survenus dans les locaux du journal satirique Charlie hebdo qui ont marqué le début d'année 2015, dans le cadre de la toute récente mise en place, par la plupart des préfectures, des «Cellules de suivi pour la prévention de la radicalisation et l'accompagnement des familles », le ministre de l'Intérieur Bernard Cazeneuve publiait une circulaire ${ }^{1}$ annonçant le lancement d'un appel d'offres pour la mise en place d'une « équipe mobile d'intervention ». C'est le Centre de prévention des dérives sectaires liées à l'islam (CPDSI) qui obtint le marché, se voyant ainsi mandaté ${ }^{2}$ pour intervenir sur l'ensemble du territoire national, dans les domaines de la prévention, du « désembrigadement », de l'accompagnement et de la formation. On a alors assisté, dans les médias, au retour massif de la notion de « manipulation mentale », que l'on pensait - à tort, puisque nous verrons qu'elle s'est en réalité perpétuée sous une pluralité d'expressions - enterrée depuis plusieurs années. Le relatif estompement de cette notion, qui fut la clé des politiques antisectes menées en France depuis les années 1970 (Esquerre, 2009), se repère dans le texte même de la loi dite «About-Picard ». Celle-ci, votée le 12 juin 2001 (par l'Assemblée nationale seulement), se proposait, pour soutenir la lutte contre les mouvements sectaires, de sanctionner leurs « atteintes aux droits de l'homme et aux libertés fondamentales $»^{3}$, sans qu'à aucun moment il n'y soit fait référence à la manipulation mentale : la création d'un « délit de manipulation mentale », en effet, d'abord envisagée, avait à ce moment-là été officiellement délaissée par les parlementaires (Bourin, 2003). Depuis lors, et durant une dizaine d'années, la notion s'est faite discrète - pour ne réapparaître, sans voile, que récemment, à la faveur du terrorisme islamiste qui

1. Circulaire NOR : INTK1504882J, du 19 février 2015 [le 7 juillet 2015, http://www.interieur.gouv.fr/Preventiondelinquance/Prevenir-la-radicalisation/Dispositions-juridiques-et-instructions-gouvernementales]

2. Le CDPSI cite la circulaire NOR : INTA1512017J, du 20 mai 2015 [le 7 juillet 2015, http://www.cpdsi.fr/]. À la date de consultation, nous n'avons pas trouvé cette circulaire sur le site du ministère de l'Intérieur [le 7 juillet 2015, http:// www.interieur.gouv.fr/]

3. La mise en « état de sujétion physique ou psychologique », " l'abus frauduleux de l'état d'ignorance ou de faiblesse », les « atteintes aux libertés et à la dignité de la personne », voire « l'atteinte à la personnalité » elle-même. Voir la Loi $n^{\circ}$ 2001-504 du 12 juin 2001 tendant à renforcer la prévention et la répression des mouvements sectaires portant atteinte aux droits de l'homme et aux libertés fondamentales [le 11 novembre 2015, http://www.legifrance.gouv. fr/affichTexte.do?cidTexte=JORFTEXT000000589924\&dat eTexte=] toucha la France. Dounia Bouzar, fondatrice du CDPSI, nous explique par exemple que le recruteur le plus actif, pour la France, est un certain Omar Omsen. «Spécialis[é] dans l'embrigadement des jeunes », Omsen «n'a guère de pouvoir militaire mais possède un immense pouvoir psychologique » (Bouzar, 2015, p. 31) : c'est ce pouvoir singulier qui en fait un recruteur efficace. La manipulation proprement dite se ferait en deux temps : d'abord la «proie » est endoctrinée par un recruteur (pour la faire adhérer à l'idéologie), puis elle est embrigadée ${ }^{4}$, c'est-à-dire amenée à reproduire « des comportements et des paroles de l'idéologie » (Bouzar, 2015, p. 69).

Nous nous proposons, dans ce travail, d'interroger ce schéma d'analyse, tel qu'il est également appliqué aux phénomènes sectaires, en déchiffrant les logiques à l'œuvre dans l'Église de scientologie. Il ne s'agit pas, bien entendu, d'amalgamer l'islamisme radical et la scientologie, mais de se demander comment un même schème explicatif (le schème manipulatoire) a pu s'imposer au point de se voir appliquer à des phénomènes aussi distincts que les sectes, la radicalisation islamiste, jusqu'aux malaises professionnels et domestiques. L'analyse du fonctionnement de la scientologie nous permettra d'en mettre au jour la mécanique de façon à montrer que nulle manipulation mentale n'y intervient. Sa dynamique s'inscrit en effet pleinement dans la logique du discours capitaliste ${ }^{5}$ qui régit nos démocraties de marché. Nous tenterons ensuite, après avoir dévoilé ce qui forme le noyau de la théorie de la « manipulation mentale », à savoir un fantasme de structure perverse, de discerner quelle part a eue, dans cet abord des phénomènes sociaux, l'invention du « pervers narcissique ». Ce n'est qu'arrivés au terme de ce parcours que nous analyserons dans toutes ses conséquences ce que le surgissement, dans la série des doctrines usant du schème manipulatoire, des figures actuelles du Père jouisseur (gourou, harceleur), nous enseigne à propos du lien social contemporain. Nous conclurons alors en interrogeant l'incidence de cette figure et de ces doctrines sur les phénomènes de radicalisation.

4. C'est donc en cohérence avec cette conception que le CPDSI propose des «stratégies » de « désendoctrinement / désembrigadement $»$.

5. «Discours » est ici à entendre au sens que Lacan (1981) donna à ce terme, celui d'armature symbolique structurant et sous-tendant le lien (social) entre les êtres parlants. 


\section{COMMENT FONCTIONNE UNE SECTE ? ASSIMILATION, TRANSFORMATIONS ET DIFFUSION DU SCHÈME MANIPULATOIRE}

En 1996 paraissait La mécanique des sectes, du Dr Jean-Marie Abgrall. Cet ouvrage, s'il ne fut pas le premier, en France, à développer une approche des logiques d'embrigadement sectaire fondée sur la notion de $«$ manipulation mentale $»^{6}$, reste certainement - peut-être par la synthèse que son auteur est parvenu à opérer entre le droit et les théories criminologiques, psychiatriques, psychanalytiques et sociologiques -, le livre « à vocation scientifique » (Dericquebourg, 2011) qui eut le plus grand retentissement pour tout ce qui touche à l'appréhension de ces phénomènes.

Abgrall amorce sa réflexion en inscrivant ce qu'il nomme les « sectes coercitives » (« SC ») dans la série des groupes - religieux, politiques, etc. - « rassemblé[s] autour d'un leader ou d'une idéologie » (Abgrall, 1996/2000, p. 15). Il précise alors que les SC se distinguent des autres groupes, d'abord par leur structure pyramidale, qui « vise à maintenir l'individu sous la contrainte » (Abgrall, 1996/2000, p. 84), ensuite par le fait que, contrairement aux nombreuses autres organisations pratiquant également la «vénération du leader», seules les SC ont pour «but ultime » «l'enrichissement de ce dernier » et « l'asservissement d'autrui » (Abgrall, 1996/2000, p. 16). Selon le Dr Abgrall, en vue d'atteindre ce double but, asservir autrui pour enrichir le leader, les sectes ont mis au point une méthode en trois temps. Le premier temps est celui du recrutement, organisé selon la séquence séduction / persuasion / fascination. Le « recruteur-séducteur » va ainsi commencer par tenter de séduire sa « cible », tout en la « détourn[ant] de la vérité » (Abgrall, 1996/2000, p. 107) : jouant sur le registre des émotions, maîtrisant ses sentiments et ses expressions de visage, il s'agit pour le recruteur de parvenir à susciter chez le « recrutable » un « processus d'identification » (Abgrall, 1996/2000, p. 108). La persuasion poursuit ce travail de détournement de la vérité en usant de méthodes de communication inspirées de la technique «ELM » (Elaboration Likehood Model) dont Abgrall précise

6. Deux ouvrages du psychologue Max Bouderlique - pour ne citer que cet auteur dont les travaux se situent dans la même zone que ceux du Dr Abgrall, à savoir « entre l'opposition aux sectes et des disciplines scientifiques » (Dericquebourg, 2011) - l'avaient précédé sur cette voie (Bouderlique, 1990, 1995). qu'elle consiste à saturer le message transmis de l'émetteur (recruteur) au récepteur (cible) avec des « facteurs irrationnels, affectifs, émotionnels, mais aussi de trucages voulus par l'émetteur » (Abgrall, 1996/2000, p. 112). Comme auparavant, il n'est pas ici question pour le recruteur de « dévoiler son projet réel », mais d'induire sa cible en erreur, quitte à enfreindre « le pacte social et tacite de la communication véritable : le présupposé de sincérité et de vérité »(Abgrall, 1996/2000, p. 114). Vient enfin le moment de fascination, contemporain de la rencontre avec le gourou ; il s'agit d'un phénomène proche de l'hypnose, fondé «sur la projection symbolique sur le gourou » (Abgrall, 1996/2000, p. 116).

Le deuxième temps du processus d'embrigadement, la persuasion coercitive, consolide les résultats du premier temps. Il vise, par diverses techniques de communication et de propagande, à déposséder entièrement l'adepte de son individualité pour l'amener à une « identification totale au gourou » (Abgrall, 1996/2000, p. 126). Il s'agit ici d'une forme de « phagocytose » (Abgrall, 1996/2000, p. 129) : une intégration dans la secte impliquant la perte de toute marque identitaire propre, de manière à faciliter la conversion de l'adepte, son « engagement définitif - "sans critique" - envers le gourou » (Abgrall, 1996/2000, p. 133). L'embrigadement sectaire se clôture par le troisième temps, le temps de conditionnement, qui «va s'appliquer selon trois modes complémentaires »: culturel, affectif et physique. Arrivé au terme du processus, l'individu, tombé dans l' « état agentique » tel que défini par Stanley Milgram (1974) dont s'inspire l'expert, est supposé accepter sans condition «le contrôle total d'une personne détenant un statut plus élevé »(Abgrall, 1996/2000, p. 148). Autrement dit, dans la théorie du Dr Abgrall, le « conditionnement mental » conduit l'individu à passer « de l'état d'autonomie totale à l'état agentique total » (Abgrall, 1996/2000, p. 148), c'est-à-dire de la plus pure liberté à l'aliénation la plus absolue.

Cette théorie va, bien entendu, être sévèrement et durablement critiquée par une partie des milieux universitaires (Anthony, 1999 ; Dericquebourg, 2011). L'idée générale de la manipulation mentale - et du lavage de cerveau - sera également largement discutée et remise en question (Champion, Cohen, 1999 ; Hervieu-Léger, 2001 ; Anthony, Introvigne, 2006). D'autres travaux académiques vont, au contraire, prendre acte des critiques adressées à la théorie d'Abgrall pour développer des approches originales $\mathrm{du}$ fonctionnement sectaire 
faisant la part belle au schème manipulatoire. Alice Norel envisage par exemple la manipulation sectaire comme une relation d'emprise prenant appui sur les défaillances narcissiques liées au vécu familial de l'adepte (Norel, 2006). Éric Péréra, dans le cadre de sa thèse soutenue à l'école doctorale Territoire, temps, sociétés et développements, déplace la question dans le champ sportif pour aborder le devenirbody builder comme un effet de l'emprise et du lien sectaire à un coach (Péréra, 2010). Un autre exemple récent, sur lequel nous allons nous attarder - il est illustratif de la persistance du schème manipulatoire dans la réflexion sur les sectes -, est fourni par la thèse de doctorat en sociologie cognitive de Romy Sauvayre (2010).

Dans son étude sur la désadhésion sectaire, Sauvayre nous présente sa position à l'égard de la manipulation mentale par le biais de ce qu'elle nomme la «métaphore de l'échoppe » (Sauvayre, 2010 , p. 82-144). La secte, nous dit-elle, si on l'envisage comme une échoppe vendant des produits divers, " est composée d'une vitrine attrayante, d'une boutique mettant à disposition ces produits et d'une arrière-boutique accessible seulement à de rares initiés » (Sauvayre, 2010, p. 84). Or, note l'auteure, dans " chacune de ces dimensions de l'échoppe se jouent des dynamiques si disjointes que la seule observation de la vitrine ne nous permettra en aucun cas d'en inférer ce qui se déroule dans l'arrière-boutique » (Sauvayre, 2010, p. 84). C'est ici, à la suite de travaux plus anciens (Lenoir, Lucas, 1998), que Sauvayre nous présente l'opposition entre les tenants de la manipulation mentale, qui tendent à extraire les sectes du domaine des croyances religieuses pour ne les envisager que sous l'angle de la tromperie, et les sociologues des religions qui abordent, au contraire, les phénomènes sectaires comme des religions minoritaires émergentes, relevant fondamentalement du champ polymorphe de la croyance et du religieux. L'opposition, selon Sauvayre, vient de ce que l'un et l'autre groupe situe ses analyses en des zones topologiques distinctes de l'échoppe : « les partisans de la théorie de la manipulation mentale, observant souvent la vitrine, la boutique et l'arrière-boutique à travers les yeux d'anciens adeptes, apporteront une vision du monde particulière », focalisée sur « l'arrière-boutique » (Sauvayre, 2010, p. 84). Tandis qu'à « l'inverse, leurs opposants - les sociologues des religions - observeront majoritairement la vitrine et la boutique de l'échoppe au sein de laquelle ils s'entretiendront avec les adeptes qui la fréquentent », sans savoir ce qui se passe dans l' arrière-boutique : « Ces sociologues pourront donc observer des personnes heureuses et épanouies, ce qui entrera en opposition complète avec la vision des partisans de la manipulation mentale » (Sauvayre, 2010, p. 84-85). D'un côté se tiendraient des sociologues dont les observations, certes justes et pertinentes, se cantonneraient néanmoins aux strates superficielles des logiques à l'œuvre dans les phénomènes sectaires. De l'autre, se situeraient les analyses usant des diverses déclinaisons de la manipulation mentale, notamment ceux de la psychologue Margaret Singer sur le « lavage de cerveau » (Singer, 2003), qui présentent, selon l'auteure, « les dynamiques inhérentes à l'arrière-boutique »: «Difficilement accessible ou visible pour tout visiteur occasionnel - tels les sociologues -, écrit Sauvayre, elle [Margaret Singer] présente une réalité relatée par nombre d'anciens adeptes » (Sauvayre, 2010, p. 91). Au fond, Romy Sauvayre, tout en relevant les excès des théories de Singer (Sauvayre, 2010, p. 87-91) et d'Abgrall (Sauvayre, 2010, p. 91-96), soutient clairement que l'approche défendue par les théoriciens de la manipulation mentale touche au cœur de la mécanique, au noyau caché (mais réel) du sectarisme - caché, du moins, aux yeux des sociologues ${ }^{7}$ qui en restent à la boutique, voire à la vitrine, soit aux couches les plus extérieures des phénomènes.

Les quelques travaux relativement récents soutenant l'idée de manipulation mentale (Norel, 2006 ; Péréra, 2010 ; Sauvayre, 2010), ajoutés aux études qui en discutent le bien-fondé (Esquerre, 2008 ; Dericquebourg, 2011 ; Mary, 2013), indiquent une certaine persistance de la doctrine de la manipulation mentale, laquelle semble être parvenue, en se diffusant par diverses voies et sous divers masques, à essaimer ses principes dans l'ensemble des débats sur les phénomènes sectaires.

Le vecteur principal de sa diffusion est le milieu des « associations antisectes », où cette doctrine rencontra immédiatement un vif succès. En se présentant comme des experts de terrain des questions sectaires, les bénévoles de ces associations sont d'abord devenus consultants auprès des grands médias où leur témoignage apparaît régulièrement, avant d'intervenir comme partenaires du monde académique. La Fédération européenne des centres de recherche et d'information sur le sectarisme (FECRIS) regroupe ainsi plusieurs associations et institutions proposant de promouvoir la lutte contre

7. L'auteure présente, à l'occasion, ces sociologues des religions comme des « sociologues militants » (Sauvayre, 2010 , p. 96), ce qui indique le peu de valeur scientifique qu'elle accorde à leur travaux. 
les dérives sectaires par un discours tantôt militant, tantôt pédagogique voire scientifique 8 . C'est dans ce cadre qu'il nous faut situer le Centre Georges-Devereux : bien qu'il ne fasse pas partie de la FECRIS, ce centre tente, lui aussi, d'articuler au discours antisectes des pratiques expérimentales et des élaborations théoriques plus académiques.

Fondé par Tobie Nathan et lié à l'Université Paris 8 , le centre fonctionne selon un dispositif de traitement et d'accompagnement des personnes « sortant de sectes » qui se compose, d'une part, d'un groupe de professionnels - cliniciens, anthropologues, historiens des religions, philosophes et sociologues - et, d'autre part, d' " au moins une personne représentant un groupe d' "experts" connaissant le fonctionnement du groupe sectaire concerné (bénévoles ou permanents de l'ADFI ou d'autres organismes de luttes contre les sectes, $[\ldots]) »$ (Nathan, Swertvaegher, 2003, p. 277). La place centrale d' « experts », « garants » des recherches et expérimentations conduites dans le centre, accordée à ces « militants antisectes » (Nathan, Swertvaegher, 2003, p. 282), peut surprendre, s'agissant d'un travail à vocation scientifique. Dans ce contexte, il n'est, en tout cas, pas surprenant de voir ressurgir la « manipulation mentale " (Nathan, Swertvaegher, 2003 , p. 42) - paradigme organisateur du militantisme antisectes -, dès le début de l'ouvrage Sortir d'une secte où nous sont présentées les méthodes du Centre. Toutefois, conformément au cadre ethnopsychiatrique qui oriente les pratiques du Centre Georges-Devereux, la problématique de la manipulation est ici repensée à partir du modèle de la sorcellerie.

Selon les auteurs, l'ensemble du processus dans lequel se sont engagés les adeptes s'origine dans « une demande de métamorphose » qui est malencontreusement tombée dans l'escarcelle d'une secte (Nathan, Swertvaegher, 2003, p. 64). Or toute secte, soutiennent-ils, au-delà des nombreux autres bénéfices attendus (prestige, plaisir sexuel, etc.), vise avant tout «[le] consentement, [le] désir » des adeptes, c'est-à-dire leur âme : « c'est de la

8. Cette double position, militante et scientifique, de la Fédération l'engage à rassembler en un unique réseau des organismes très disparates : parmi les correspondants de la FECRIS, l'on trouve aussi bien l'International Cultic Studies Association (ICSA), qui publie une revue scientifique, l'International Journal of Cultic Studies [le 12.11.2015: http://www.icsahome.com/elibrary/ijcs], que « Secticide », une association militante de lutte contre les dérives sectaires. "capture" de cette âme que des organismes aux intentions malveillantes attendent des bénéfices » (Nathan, Swertvaegher, 2003, p. 57). Pour mettre en œuvre cette « capture d'âme », les groupes sectaires mobilisent, au détriment des personnes qui se sont laissé séduire, une «puissance étrange et [...] difficile à penser » : « la promesse d'être initiées à de nouvelles connaissances, de participer à un rite ignoré du commun et de bénéficier du pouvoir qui en découle » (Nathan, Swertvaegher, 2003, p. 60). La mécanique sectaire est donc, ici, proche du processus initiatique traditionnel. À une différence cruciale près, rappellent les auteurs : « les initiations traditionnelles sont des procédures de fabrication qui ne transforment les personnes qu'en ce qu'elles étaient déjà : l'homme qui était bambara de naissance deviendra, du fait de l'initiation [...] un bambara et rien d'autre qu'un bambara » (Nathan, Swertvaegher, 2003, p. 122). L'initiation sectaire, au contraire, tente de « transformer des personnes en ce qu'elles n'auraient aucune chance d'être si elles n'avaient pas rencontré le groupe ». Autrement dit, l'initiation traditionnelle se voue à produire des « hommes à l'ancienne », tandis que les groupes sectaires «veulent toujours produire un homme nouveau » (Nathan, Swertvaegher, 2003, p. 123). Pour «fabriquer » cet homme nouveau « conforme au modèle proposé par le groupe », l'initiation sectaire fonctionnera « à l'inverse d'un "enseignement", non pas par persuasion, mais par imposition, selon une "logique traumatique", qui exclut l'explication » et le sens, lequel est renvoyé au terme du processus initiatique (Nathan, Swertvaegher, 2003, p. 120-121). Mais, telle est la spécificité des sectes, celui-ci est interrompu avant son terme, ce qui fait que le sens n'advient pas et que l'adepte, en lieu et place de l'initiation à laquelle il aspirait, se voit confronté à une brutale opération de conversion.

La conversion est, selon les auteurs, « une procédure de désafiliation / réafiliation », « un événement révolutionnaire [exigeant] du sujet un abandon radical de tout ce qui pourrait le rattacher au passé révolu » (Nathan, Swertvaegher, 2003, p. 125) : groupes d'origine, famille, travail, etc. Cet état de « désafiliation », d'ajournement indéfini du sens consécutif à la promesse d'initiation non réalisée est très exactement le nœud de l'opération sectaire, selon les auteurs - c'est cette « promesse, écrivent-ils, toujours en attente d'être réalisée [qui crée] en vérité l'addiction » (Nathan, Swertvaegher, 2003, p. 60). La notion de manipulation mentale, loin de disparaître lors de son passage par l'ethnopsychiatrie, y a, au contraire, trouvé matière à 
démontrer sa grande plasticité : relue à l'aune de la sorcellerie, elle devient, chez Nathan et Swertvaegher, une modalité d'emprise totale sur les adeptes grâce au « rapt » de leur âme, promise à servir le groupe et le gourou.

Notons enfin, pour terminer ce survol des diverses transformations qui ont permis la perpétuation du schème manipulatoire, que celui-ci s'est à la fois diffusé, nuancé et discrètement installé dans le champ de l'analyse des sectes lorsqu'il trouva à s'établir dans le discours politique. La jonction entre les discours académique et politique se fit par le truchement des travaux d'Anne Fournier - ancienne élue locale (à Villeneuve-Saint-Georges, entre 1989 et 1995), qui devint, à partir du milieu des années 1990, experte auprès de la Mission interministérielle de lutte contre les sectes (MILS), puis de la Mission interministérielle de vigilance et de lutte contre les dérives sectaires (Miviludes). Sa légitimité académique (elle est agrégée d'histoire et diplômée de l'Institut d'études politiques de Paris) lui a certainement permis d'inscrire durablement, au cœur des réflexions parlementaires portant sur la question des sectes, sa version originale du paradigme manipulatoire dont on découvre le cadre général dans son ouvrage co-écrit avec Michel Monroy (Fournier, Monroy, 1999). L'expression «manipulation mentale » y laisse place à celle, plus floue et moins chargée idéologiquement, d' " emprise sectaire », en laquelle se perpétuent pourtant non seulement la même idée de manipulation mentale, mais aussi la même séquence en trois temps que l'on trouvait dans le conditionnement coercitif abgralien : séduction, transformation de la personnalité et reconstruction (d'une nouvelle personnalité). La notion d'« emprise » donnera lieu, à son tour, à divers travaux publiés dans des revues académiques (Diet, 2004 ; Maes, 2002 ; 2006) $)^{9}$. Avec l'introduction et la diffusion de cette notion dans le débat sur les sectes, le poids de la situation sectaire tendra à se déplacer du gourou vers le groupe, mais sans pour autant se débarrasser complètement du gourou, qui reste le pivot de l'opération. C'est ainsi, par exemple, que Chouvier et Morhain (2007), s'appuyant sur les élaborations kleiniennes concernant les positions shizo paranoïde et dépressive, parleront d'une " position sectaire », un processus régressif susceptible d'être mis en place par tout groupe lorsque surviennent des

9. J.-C. Maes, fondateur de l'association « SOS Sectes » et, par ailleurs, l'un des premiers à avoir employé cette notion (Maes, 1999), dévoilera clairement les liens entre l'emprise et la manipulation mentale abgrallienne (Maes, 2010). situations menaçant sa cohésion et son identité. Il arrive alors qu'un fonctionnement pervers s'impose à l'intérieur du groupe, qui peut ainsi progressivement se mettre à développer "sciemment l'assujettissement de ses membres au profit des désirs du chef et de ses acolytes » (Chouvier, Morhain, 2007, p. 26). Ce sont ces échos relativement atténués de la manipulation mentale qui résonneront encore dans les rapports publiés par les commissions interministérielles traitant de la question sectaire ${ }^{10}$.

Dès son premier rapport, publié en 2003, la Miviludes introduit en effet la manipulation mentale sous deux nouvelles dénominations, la « déstabilisation mentale » et la «sujétion mentale » (Miviludes, 2003, p. 6-7, 10). Le rapport de 2004 nous indique l'« une des définitions possibles pour qualifier un groupe "d'organisation sectaire", quels que soient sa taille et son objet », à savoir « la capacité qui est la sienne de modifier la personnalité de ses adeptes en vue de favoriser l'allégeance inconditionnelle au clan et à son gourou ». L'ensemble des pratiques du groupe à l'égard de l'adepte vise, selon la commission, un unique objectif : «le façonner selon le modèle imposé de l'adepte docile et entièrement disponible, asservi à la loi du groupe » (Miviludes, 2004, p. 62). Nous retrouvons donc, dans ce rapport, divers éléments déjà présents chez Abgrall : non seulement la sujétion mentale et l'aliénation de l'adepte au groupe et à son leader, mais aussi l'idée d'une " emprise sectaire, construite sur un mode pervers » (Miviludes, 2004, p.63). Une modification apparaît toutefois, dès 2004, concernant l'adepte : contrairement à la théorie abgrallienne, où il apparaît entièrement passif, la commission interministérielle note qu'il est en réalité, comme le soutient Anne Fournier (2000, 2010), un « acteur consentant» (Miviludes, 2004, p. 63). Dans le rapport publié en 2006, ce consentement actif est analysé à partir des travaux de Raymond Boudon (1993) : pour obtenir le consentement de l'adepte, nous dit la commission, la secte l'engage dans un enchaînement d'acquiescements successifs qui lui apparaîtront « comme des choix déterminés et conscients » (Miviludes, 2006, p. 16). Il n'en reste pas moins que ce qui nous est présenté comme la visée du processus demeure l'emprise et la «mise en état de sujétion » : pour la Miviludes, comme pour Abgrall, il s'agit avant tout de soumettre totalement l'adepte. Ces considérations, sur lesquelles

10. En 1998 était créée la Mission interministérielle de lutte contre les sectes (MILS), qui s'est vue remplacée, en 2002, par la Mission interministérielle de vigilance et de lutte contre les dérives sectaires (Miviludes). 
nous reviendrons, s'appliquent-elles à la scientologie, le modèle-étalon des sectes manipulatrices ?

\section{DYNAMIQUE SECTAIRE, CYCLE PULSIONNEL ET LOGIQUE CONSUMÉRISTE}

Le parcours initiatique des adeptes de l'Église de scientologie se décompose en deux grandes étapes. La dianétique forme le premier segment de l'initiation scientologue. Il s'agit d'une méthode de développement personnel fondée sur une technique psychothérapeutique, l'« audition de dianétique », fortement inspirée de la cure cathartique de Freud et Breuer (1895/2005). En dianétique, un « audité », mis sous hypnose, répond aux questions d'un « auditeur », qui l'engage à remonter le fil de sa vie, traumatisme après traumatisme, jusqu'à une semaine avant sa fécondation, pour atteindre le premier incident générateur de ses troubles (le «basiquebasique ») de façon à le « décharger ». Le second segment du parcours initiatique - «l'audition de scientologie »- engage l'adepte sur la voie de ses innombrables vies passées, pour le mener à l'état de « Thétan opérant », ou « OT ». Le Thétan opérant est une entité parfaite, immunisée contre toutes les maladies, un esprit supérieurement puissant qui est capable de modifier à sa guise la structure moléculaire de la réalité. C'est cette figure omnipotente, centrale dans la mythologie scientologue - et non pas quelque gourou tyrannique -, qui suscite l'engagement sectaire en polarisant le désir des adeptes.

On peut repérer un cycle en trois temps qui dynamise l'expansion mondiale du mouvement scientologue. Dans le premier temps, le désir des adeptes est causé par un objet (le Thétan opérant) promu en place d'idéal par le groupe. Lors du deuxième temps, ce désir enclenche le cycle pulsionnel qui pousse l'adepte à consommer les produits vendus par la secte pour atteindre l'état de «OT». Et enfin, troisième temps, une fois le produit consommé, l'adepte s'expose à la rencontre ponctuelle et traumatique de cette modalité particulière de l'extase que Ron Hubbard nomma le « rêve spermatique » (Hubbard, 1950/2003). Durant ces expériences profondément troublantes, proches de celles des toxicomanes, les adeptes se sentent flotter dans un liquide chaud, envahis de sensations indicibles. Ce sont ces sensations ineffables, disent-ils, qui potentialisent, d'une certaine façon, leur dépendance aux produits vendus par la secte : rendus « accro ${ }^{11}$ »

11. Selon l'expression employée par Mona Vasquez, qui fut elle-même adepte de la secte.
(Vasquez, 2004, p. 23), ils n'aspirent qu'à en consommer plus.

Ce qui nous intéresse ici, c'est que le fonctionnement libidinal (en trois temps) de la secte est très exactement calqué, non pas sur le processus repéré par Abgrall, mais sur celui qui met en mouvement le marché mondial de la consommation.

Dans ce qu'on pourrait appeler le premier temps du processus consumériste, un produit de consommation, promu à une certaine place parmi les idéaux d'une culture (ou d'un groupe) par la publicité et avec la complicité des médias, cause le désir des sujets-consommateurs, suscite la demande et mobilise la pulsion. C'est le publiciste, Edward Bernays ${ }^{12}$, qui nous a révélé le fonctionnement intime de ce premier temps. Il montre, dans son ouvrage de 1928, Propaganda : comment manipuler en démocratie?, d'une part, que nos choix ne sont pas guidés par des décisions conscientes et rationnelles, mais qu'ils sont déterminés par nos désirs inconscients ; et, d'autre part, que nos décisions, loin de dévoiler directement l'objet de notre désir, désignent en réalité une représentation (métaphorique, déplacée) de cet objet. Bernays (1928/2007) avait en outre saisi, en une sorte d'anticipation de Lacan, que «Le désir de l'homme, c'est le désir de l'Autre » (Lacan, 1975, p. 11) : ce dont on pense avoir besoin, c'est en réalité ce que l'Autre (l'Autre du social ou des médias) nous incite à désirer, via les divers discours qui donnent corps aux idéaux d'une culture.

Bernays (1928/2007) en tira évidemment la seule conclusion logique, à savoir qu'il n'y a pas, chez l'homme, de besoins « naturels ». Nos besoins, puisqu'ils sont médiatisés par le langage, sont en effet nécessairement marqués d'un moins, d'une faille, donc d'un manque qui alimente le désir. $\mathrm{La}$ consommation n'a ainsi rien à voir avec le besoin : elle est affaire de désir (inconscient). De fait, le travail d'un publiciste ne peut se réduire à vanter les caractéristiques objectives d'un produit supposé combler adéquatement un besoin réel. Son travail consistera plutôt à tenter d'élever une quelconque marchandise à la dignité d'un objet du désir, pour susciter chez le client la demande qui enclenchera le cycle pulsionnel, c'est-à-dire le besoin de consommer. Bernays développera alors des stratégies inédites dont le principe est très bien résumé par Normand Baillargeon :

12. Edward Louis Bernays est le fils d'Anna, la sœur de Sigmund Freud, et d'Eli Bernays, son beau-frère (le frère de son épouse Martha). 
« au lieu de simplement décrire en les vantant les caractéristiques d'un produit, d'une cause, ou d'une personne, cette nouvelle forme de publicité $[\ldots]$ les associe à quelque chose d'autre, que le public, [selon] Bernays, ne peut manquer de désirer » (Baillargeon, 1928/2007, p. 9). C'est ainsi que procède la scientologie : elle élève n'importe lequel de ses produits à la fonction d'objet du désir en en faisant l'une des marches nécessaires pour pouvoir atteindre la position prestigieuse du Thétan opérant.

Le deuxième temps du processus consumériste se nourrit du fonctionnement même de la pulsion, tel que Freud l'a repéré. Comme on le sait, la particularité de la pulsion est de ne pas avoir d'objet spécifique. Elle trouve à se satisfaire dans le ratage de l'objet visé, dans son contournement, qui ne peut que raviver la demande (d'une autre marchandise), ainsi que la remobilisation de l'appareillage pulsionnel en direction d'un nouvel objet (Lacan, 1973). C'est ce fonctionnement qui assure le succès du marché de la consommation : la pulsion n'ayant pas d'objet spécifique, aucune marchandise ne peut la satisfaire. Autrement dit, aucune marchandise ne peut étancher la demande, ce qui rend le mouvement pulsionnel inextinguible : chaque ratage de l'objet a pour effet de relancer la flèche de la demande. Quant au troisième temps, il est, en réalité, le premier temps d'un nouveau cycle, comme Karl Marx l'avait finement repéré : « l'acte final de la consommation $[\ldots]$, se situe, à vrai dire, en dehors de l'économie, à moins qu'il réagisse sur le point de départ et inaugure à nouveau tout le processus » (Marx, 1835-1867/1994, p. 454-455). La réussite (financière) de la scientologie est ainsi proche de celle des grandes enseignes internationales : elle est directement liée à sa capacité de vendre des produits qui, tout comme certaines grandes marques de l'industrie, rendent ses clients dépendants. C'est donc, non pas en raison de techniques de manipulation mentale, mais parce que l'image idéalisée du Thétan opérant cause leur désir, que les sujets s'engagent dans le cycle aliénant de la consommation des produits vendus par la secte.

Pour chacun des adeptes du mouvement, l'impératif moral majeur est l'optimisation de son propre « potentiel », voie royale vers sa survie individuelle, c'est-à-dire vers sa propre immortalité dont est supposée dépendre, secondairement, celle du groupe et de l'humanité. L'ex-scientologue Jenna Miscavige Hill le confirme en définissant la scientologie comme « une philosophie, un programme d'aide personnelle permettant d'aboutir à une meilleure connaissance de soi et offrant la possi- bilité d'optimiser au mieux son propre potentiel » (Miscavige, Pulitzer, 2013, p. 17). « Chaque victoire, souligne-t-elle par ailleurs, n'était en réalité qu'un gain personnel » (Miscavige, Pulitzer, 2013, p. 136). On voit ici combien le moi, la personne, l'ego, sont au cœur du processus. En scientologie, chaque adepte se voue, avant tout, à faire un travail sur soi. Il poursuit, comme le dit si bien Miscavige, ses gains personnels ${ }^{13}$. Le but de tout scientologue est de se «clarifier» (Hubbard, 1951/2003), c'est-à-dire de se libérer de ses « engrammes » (Hubbard, 1951/2003), de façon à accéder à la vie éternelle. Mais l'immortalité, selon la doctrine scientologue, ne pourra s'actualiser, pour chacun, que lorsque l'ensemble des êtres humains seront devenus « clairs », c'est-à-dire lorsque toute l'humanité sera convertie à la scientologie. D'où la nécessité, pour tout adepte, de consacrer une partie de son temps au prosélytisme, mais non pas tant pour sauver les personnes recrutées, que pour se sauver soi-même. L'aiguillon principal qui pousse l'adepte à s'engager toujours plus loin dans le processus initiatique de la secte, est ainsi son intérêt personnel. C'est cet intérêt personnel qui aboutit secondairement et comme sous l'effet d'une force providentielle d'une « main invisible » (Smith, 1776/1999), à l'intérêt de tous. Tout se passe, au final, comme si la scientologie avait repris à son compte et appliqué à la lettre la préconisation libérale d'Adam Smith lorsqu'il affirma que le bien public dépendait du fait que chacun ne poursuive que son seul intérêt égoïste. On comprend, ici, que la scientologie ne nous révèle pas une violence étrangère à notre modernité. Sa violence est issue des mêmes «eaux froides du calcul égoïste » (Marx, Engels, 1848/1989, p. 19) que le capitalisme. C'est pourquoi cette secte, qui applique strictement les principes d'Adam Smith, nous dévoile, pour ainsi dire, les logiques libérales sauvages qui président au mouvement même de l'Occident contemporain. Dès lors, est-il possible, comme le supposent les experts, de débusquer les indices de l'existence d'une autorité toute-puissante manœuvrant en coulisses les agissements des adeptes?

13. L'on voit nettement ici combien la scientologie propose une religiosité en phase avec son époque : elle promeut, à la façon de l'ésotérisme $\mathrm{New}$-Age, une religiosité orientée sur soi et sur ce monde-ci (Hervieu-Léger, 2010). Comme dans tous « les mouvements spirituels contemporains de terrain chrétien aussi bien que dans la vaste mouvance des nouveaux mouvements religieux de genre ésotérique », le primat est ici accordé « à l'expérience personnelle que chacun mène selon sa voie propre et qui doit lui permettre d'atteindre à l' accomplissement de lui-même » (Hervieu-Léger, 2010, p. 23). 
Pour tirer au clair ce point précis, nous devons lire de nouveau ce que nous en dit Jenna Miscavige Hill, qui a passé la majeure partie de sa vie en scientologie. La jeune femme, qui est entrée encore enfant dans la secte, nous apprend que c'étaient essentiellement les autres enfants, un peu plus âgés, qui prenaient soin des plus jeunes. En effet, toute relation asymétrique (à l'adulte) était à ce point estompée que, dans leur école, « [i]l n'y avait ni notes, ni bulletin scolaire, et l'enseignant ne cherchait pas à s'imposer comme maître à penser de sa classe » (Miscavige, Pulitzer, 2013, p. 56). Elle précise d'ailleurs que l'enseignant n'en était pas un : le terme « enseignant» n'était pas utilisé, et chacun se devait d' " apprendre [les matières "classiques"] par [ses] propres moyens » (Miscavige, Pulitzer, 2013, p. 79). Même lorsqu'un nouveau règlement institua une discipline « militarisée », les mauvaises notations ne furent jamais le fait d'une quelconque autorité abusive, mais d'un autre enfant, membre du groupe. Ainsi, dans l'univers sans autorité, sans asymétrie, donc strictement horizontal de la scientologie, c'est un principe d'auto-surveillance, une pression immanente au groupe de pairs (Miscavige, Pulitzer, 2013, p. 63), qui suscite le sentiment d'oppression. C'est pourquoi nul gourou n' apparaît ici responsable de l'aliénation puissante des adeptes : tous, enfants et adultes, progressent et s'engagent de plus en plus loin dans leur initiation, non pas pour obéir aux ordres d'une hiérarchie pyramidale, mais pour coller au plus près de leur idéal. Le moteur de l'initiation est alors, plus exactement, la quête de leur intérêt égoïste, fondée sur une identification (narcissique) au Moi-idéal. Miscavige, entre autres, le révèle : « depuis mon arrivée à la Flag ${ }^{14}$, j'avais rencontré de plus en plus de personnes très avancées dans leur cursus, et certaines étaient très rapidement devenues pour moi des modèles à suivre. Je voulais ce qu'ils avaient : plus de connaissances, être plus proche de la liberté totale » (Miscavige, Pulitzer, 2013, p. 133).

\section{STRUCTURE DU FANTASME}

Cette « liberté totale » dont Miscavige entrevoyait le reflet chez les adeptes plus avancés contredit le postulat de la mise en état de sujétion défendu par les experts. Les manipulations sectaires ne visent-elles pas, en effet, à enfermer les adeptes dans l'« état

14. «Flag est le quartier général de la Scientologie. Cette dénomination est la contraction du mot Flagship ou bateau amiral car il était au départ établi sur le bateau Appolo » (Ariès, 1998, p.33). agentique » ? Citant Milgram, Abgrall décrit ainsi cet état résultant de l'emprise sectaire : « dans ce cas, il [le sujet réduit à l'état agentique] ne s'estime plus responsable de ses actes. Il voit en lui-même un simple instrument destiné à exécuter les volontés d'autrui » (Abgrall, 1996/2000, p. 148).

Voilà des termes forts, dont le moins que l'on puisse dire est qu'ils nous envoient bien loin de l'expérience sectaire dont Jenna Miscavige témoigne. N'a-t-on pas, en revanche, l'impression de lire, dans cette citation empruntée à Milgram, la description fidèle de la situation de Truman (héros du film The Truman Show, 1998) et de Néo (héros de la trilogie Matrix, 1999), deux personnages de fictions cinématographiques réalisées à la fin des années 1990 ? L'un et l'autre, en effet, vivant enfermés dans des univers cloisonnés et artificiels, prisonniers passifs soumis aux conditionnements, manœuvres et manipulations d'un pouvoir tout-puissant dont l'unique horizon semble être de jouir d'eux, ne ressemblent-ils pas à s'y méprendre aux adeptes de sectes tels que nous les décrivent les études fondées sur la manipulation? Autrement dit, si ces analyses de l'état de sujétion mentale ne nous apprennent pas grand-chose de l'expérience sectaire proprement dite, on a, par contre, le sentiment qu'elles pourraient bien receler un noyau fantasmatique, s'exprimant de façon prégnante dans le monde contemporain et infiltrant dès lors massivement certains thèmes - et plus particulièrement le thème de la manipulation - dans la culture, le cinéma ou la littérature. Ce fantasme serait alors propice, au final, à nous en dire long, non pas sur l'expérience sectaire, mais sur l'expérience subjective.

Rappelons que le fantasme a très précocement retenu l'attention de Freud qui l'évoquait déjà dans une lettre à Fliess du 6 avril 1897 (Freud, 2006, p. 297). Dans sa définition intuitive, il renverrait à « l'idée d'un scénario imaginaire soutenant la rêverie diurne, parfois l'activité sexuelle, en nous permettant, du moins nous le croyons, d'échapper à la réalité »(Castanet, 2002, p. 291). Lacan nous enseigne pourtant qu'il n'en est pas exactement ainsi : loin de nous permettre d'échapper à la réalité, comme le font les rêveries, le fantasme, selon lui, c'est au contraire ce qui donne à la réalité son cadre ${ }^{15}$. Freud fut d'ailleurs le premier à avoir repéré que les souvenirs traumatiques tirent leur

15. La réalité est ce « champ [qui] ne fonctionne qu'à s'obturer de l'écran du fantasme » et qui « ne se soutient que de l'extraction de l'objet $a$ qui pourtant lui donne son cadre » (Lacan, 1959/1966, p. 553-554). 
force et leur valeur, non d'un événement de la réalité, mais d'une scène fantasmatique ${ }^{16}$.

Dès 1897, il envisage les fantasmes comme des constructions signifiantes dont le principal effet est de remanier et d'introduire de la distance à l'égard des expériences vécues. Freud reconnait ainsi qu' " une face inconsciente pousse l'homme à remodeler son expérience et son souvenir et y [voit] l'effet d'un désir premier » (Castanet, 2002, p. 292). Les fantasmes se présentent dès lors comme l'effet de ce désir archaïque, comme la matrice où s'élaborent les désirs actuels. "Ce sont eux qui transforment les perceptions et les souvenirs, [et] qui sont à l'origine des rêves, des lapsus et des actes manqués »(Castanet, 2002, p. 292). Ainsi, le fantasme oriente toutes les activités du sujet, s'exprime dans ses rêves diurnes et cherche à s'actualiser « de façon déguisée, par les choix professionnels, relationnels, sexuels et affectifs du sujet » (Castanet, 2002, p. 292). Mais il y a un second aspect du fantasme, tout aussi important, quoique peut-être plus fondamental.

Il résulte de l'une des expériences majeures dans lesquelles le fantasme s'enracine, à savoir ce moment anhistorique, quasi mythique, où la mère, que nous appellerons avec Lacan le « grand Autre » (le premier Autre, du moins), par ses allées et venues, par l'alternance de sa présence et de son absence, s'est révélée désirante. Ce moment pivot est celui par lequel s'est introduit le soupçon que l'Autre est manquant. C'est précisément en ce point, là où menacerait de se révéler la castration - l'inconsistance - de l'Autre, qu'intervient le fantasme, dont « la fonction [...] est [précisément] de servir d'écran pour cacher cette inconsistance » (Žižek, 1990, p. 152). Le fantasme, lorsqu'on l'envisage selon cet autre versant, est donc un «montage ayant pour fonction de convoquer, et simultanément de faire écran à ce qui est radicalement insupportable pour un sujet ([à savoir] ce que Lacan nomme le "réel") » (Abelhauser, 2010, p. 7). Le fantasme, donc, dans le même mouvement, mobilise et masque le réel. Autrement dit : si le fantasme, par un bord, structure notre réalité, s'il est notre porte d'entrée

16. Lorsqu'il abandonna ses «neurotica » (Freud, 2006, p. 334), en septembre 1897, Freud délaissa l'hypothèse selon laquelle les traumatismes étaient liés à des scènes originaires réelles, pour situer leur source en des fantaisies nées d'expériences ultérieures qui « retournent puiser dans l'enfance » (Freud, 2006, p. 335) la matière signifiante - la « liaison de mot» (Freud, 2006, p. 430), dira-t-il plus tard dont ils se constituent. dans la réalité, c'est au prix - second aspect - d'un recouvrement du réel ${ }^{17}$, d'un voilement de cette jouissance dont le manque fait l'Autre inconsistant (Lacan, 1960/1966, p. 819-820).

\section{L'AUTRE JOUISSEUR ET SON DOUBLE, LA VICTIME}

La doctrine de la manipulation mentale, en interprétant les phénomènes sectaires comme les conséquences des intentions malveillantes de l'Autre, de son vouloir tout-puissant, en suggérant sa jouissance, donne donc consistance à ce grand Autre. Elle le complète du pouvoir qu'il n'a pas. C'est en ce sens, parce qu'elle vise à soutenir la complétude de l'Autre, que cette doctrine a la structure du fantasme : le réel (ce quelque chose qui décomplète l'Autre et le fait inconsistant) est escamoté par l'apparition, au premier plan des discours, de la figure fantasmatique du manipulateur / harceleur. Mais à bien lire ce qu'implique la doctrine de la manipulation mentale, on découvre rapidement qu'il ne s'agit pas simplement de donner de la consistance à l'Autre. Il s'agit aussi et surtout, dans le même mouvement, de passiver le sujet, de le rendre totalement impuissant, de façon à le livrer à la jouissance de l'Autre, à son impérieuse voracité, encore une fois comme dans Matrix. Voilà bien en quoi cette doctrine n'a pas simplement la structure du fantasme, mais la structure du fantasme pervers. $\mathrm{La}$ passivité complète des adeptes tels que la doctrine nous les décrit, proche en tout point de l'immense passivité des humains dans Matrix, met en scène « le fantasme qui soutient notre expérience de sujets actifs [...] - elle fournit [cette passivité] le fantasme pervers ultime, l'idée qu'en définitive nous sommes des instruments de la jouissance de l'Autre (de la matrice), et que, comme dans une batterie, nous voyons sucée notre substance vitale » (Žižek, 2010, p. 349).

Ce fantasme consiste, dans sa mise en scène, à se faire l'instrument passif de la jouissance de l'Autre. Il sous-tend et soutient l'expérience du sujet actif contemporain, c'est-à-dire du sujet de la postmodernité, celui qui est tenu d'advenir comme sujet sans disposer des repères symboliques qui balisaient jadis le cheminement subjectif, au temps des cultures fondées sur l'autorité. Arrivé à ce point, on peut déjà faire une première remarque :

17. Le réel n'est pas ici à entendre dans le sens de « réalité », mais dans son sens lacanien, comme le rebut de l'opération langagière : ce qui, de structure, échappe au symbolique (objet $a$ ). 
lorsque le tiers - celui qui prenait autrefois en charge la question des origines, et qui se proposait comme support de l'autorité -, vient à manquer, ou à vaciller en perdant de sa légitimité, il peut se voir remplacé par la figure fantasmatique féroce du Père-jouisseur. C'est peut-être bien ce qui explique le succès, depuis trois décennies, de cette série de grandes figures persécutrices : le « pervers narcissique », le « harceleur » et le « gourou-manipulateur ». Détaillons chacune de ces trois versions de la malignité de l'Autre, afin d'en mesurer la profonde familiarité.

La notion de "perversion narcissique », catégorie nodale et fondatrice de la série ${ }^{18}$, a été inventée et systématisée par le Dr Paul-Claude Racamier, au tout début des années $1990{ }^{19}$. Nous en retrouvons historiquement une forme d'anticipation dans les travaux de Dupré (1905), l'inventeur du mythomane. C'est à ce praticien éminent que l'on doit l'introduction, dans la clinique psychiatrique moderne, dès l'orée $\mathrm{du} \mathrm{Xx}^{\mathrm{e}}$ siècle, certaines des idées les plus tenaces touchant à la mystification et à la simulation : les mythomanes, puis, 70 ans plus tard, les pathomimes du Dr Corraze (1976), furent en effet durablement soupçonnés de manipuler les médecins pour le simple plaisir de les posséder, autrement dit pour jouir à leurs dépens. Engluées dans l'imaginaire, ces catégories, ainsi que d'autres qui ont éclos au xxe siècle, pervertissent la clinique en l'interprétant à partir de jugements moraux. Il n'y a donc pas lieu de s'étonner que le pervers narcissique, héritier de cette clinique, semble uniquement mû par le besoin insatiable de dominer l'autre, quitte à le réduire à l'état d' " ustensile », d'objet interchangeable entièrement soumis à ses menées. « Maltraité, sadisé, [...] maîtrisé », l'autre, entre ses mains, n'est « rien de plus et rien de moins qu'une marionnette » (Racamier, 2012/2013, p. 36). Les deux versants de la situation sont indissolublement liés, comme l'avers et l'envers d'une pièce. D'un côté se tient le pervers narcissique : un être de « prédation » (Racamier, 2012/2013, p. 36)

18. La Miviludes reconnaît d'ailleurs non seulement la préséance de la question perverse, mais la familiarité de ces notions, lorsqu'elle écrit, dans son rapport de 2006 : «L'expression [d'“emprise sectaire”] s'est généralisée parce que les psychologues ont beaucoup avancé sur la notion d'emprise perverse ou de harcèlement moral ».

19. Le livre de Racamier, Les perversions narcissiques (2012/2013), reprend l'essentiel de sa théorie du pervers narcissique telle qu'il la décrit dans les chapitres 9 et 10 de son ouvrage Le génie des origines (1992) - c'est pourquoi nous ne citerons que son ouvrage de 2012, qui résume bien sa thèse. qui n'est que pure volonté de puissance, pur agir dont le trognon de pensée ne se préoccupe que de manipuler, dominer et détruire celui (ceux) qu'il prend pour objet(s). Sur l'autre face se situe la « proie » (Racamier, 2012/2013, p. 36) du pervers narcissique, son « objet ustensilaire » (Racamier, 2012/2013, p. 36) : sa marionnette.

C'est à partir de ce binôme (pervers/partenaire passif) et de cette trame de fond que M.-F. Hirigoyen va reprendre, pour l'adapter au contexte français, la réflexion (initialement anglo-saxonne ${ }^{20}$ et suédoise ${ }^{21}$ ) sur ce qu'elle nommera le « harcèlement moral ». Dans ses deux principaux ouvrages sur la question (Hirigoyen, 1998/2001, 2001/2013), le harcèlement (domestique et professionnel) est directement lié à la figure du « pervers narcissique » (Hirigoyen, 1998/2001, p. 149-164, 2001/2013, p. 345-349). L'auteur en présente les qualités en deux temps : en tant que pervers (au sens moral de perversité, comme chez Racamier, 2012/2013, p. 51), la conduite de cet être «implique une stratégie d'utilisation puis de destruction d'autrui, sans aucune culpabilité » (Hirigoyen, 1998/2001, p. 149) ; et en tant que narcissique, il « est une coque vide qui n'a pas d'existence propre ; c'est un "pseudo", qui cherche à faire illusion pour masquer son vide» (Hirigoyen, 1998/2001, p. 154). Or c'est précisément en cherchant à remplir ce vide que cet être sans substance va, selon l'auteur, basculer dans la perversion : «Le Narcisse, n'ayant pas de substance, va se brancher sur l'autre et, comme une sangsue, essayer d'aspirer sa vie. Étant incapable de relation véritable, il ne peut le faire que dans un registre "pervers", de malignité destructrice » (Hirigoyen, 1998/2001, p. 154). Ici, comme chez Racamier, le pervers narcissique est un être foncièrement inquiétant : mû par la malveillance, l'envie de nuire et une agressivité structurale, il nous est dépeint comme un "vampire » qui a «besoin de se nourrir de la substance de l'autre » (Hirigoyen, 1998/2001, p. 154). Le partenaire, précise Hirigoyen, est «manipulé » et mis en état de « sidération » pour être purement et simplement « détruit » : « [il] n'existe pas en tant que personne mais en tant que support d'une qualité que les pervers essaient

20. Le psychiatre américain Carroll Brodsky (1976) a repéré les phénomènes d' « harassment » dès le début des années 1970 , et en a rapidement publié un ouvrage.

21. Après avoir publié, dans les années 1980, plusieurs articles et enquêtes sur le «mobbing », c'est-à-dire sur la violence liée aux phénomènes de groupes, Heinz Leymann publie, en Suède, un ouvrage de vulgarisation en 1993, qui sera peu après traduit en français (Leymann, 2002). 
de s'approprier. Les pervers se nourrissent de l'énergie de ceux qui subissent leur charme » (Hirigoyen, 1998/2001, p. 157). Le pervers narcissique est donc animé de la même voracité insatiable que la Matrice du film des Wachowsky : tous deux ont « besoin de la chair et de la substance de l'autre pour se remplir » (Hirigoyen, 1998/2001, p. 157). Mais à la différence de la Matrice wachowskienne, qui est une mécanique aveugle (sans affect), les pervers narcissiques, en revanche, détruisent l'autre avant tout parce qu'ils « jouissent de la souffrance » qu'ils infligent (Hirigoyen, 1998/2001, p. 159). Nous pouvons résumer la théorie de Hirigoyen en une courte phrase : le harcèlement moral désigne une situation (familiale, professionnelle) dans le cadre de laquelle un Autre jouisseur vampirise une victime passive via de sournois mécanismes de domination manipulatoire. L'on voit ici combien le harceleur ressemble au gourou des doctrines antisectes. Comme le gourou, le harceleur use lui aussi de l'« emprise », cette « action insidieuse » qui « retire tout sens critique » et « peut aller jusqu'à la captation de l'esprit de l'autre, comme dans un véritable lavage de cerveau ». En conséquence, « la victime est [alors supposée] prise dans une toile d'araignée, tenue à disposition, ligotée psychologiquement, anesthésiée »(Hirigoyen, 1998/2001, p. 113). Pourquoi ces figures inquiétantes ont-elles commencé à peupler massivement l'imaginaire occidental dans le courant des années 1990 ? Pour le saisir, il nous faut envisager ces diverses entités (pervers narcissique, harceleur et gourou), aussi tentaculaires que nébuleuses, comme autant d'indices de la dégradation contemporaine de la fonction paternelle.

Dès 1938, Lacan s'alarma des conséquences, sur la famille et le sujet, du « déclin social de l'imago paternelle »(Lacan, 1938/2001, p. 60). Ce n'est pourtant que dans les années 1980 et 1990 que ce déclin commença à manifester ses effets dans le vivre-ensemble, par le truchement des mutations dans l'organisation et le management d'entreprise. Les structures hiérarchisées firent alors place aux systèmes en réseau (Boltanski, Chiapello, 1999/2010, p. 109-118), les cadres devinrent des managers, voire des coachs (Boltanski, Chiapello, 1999/2010, p. 119-124), tandis que chaque salarié, via les principes du «management participatif » (Marzano, 2008/2010, p. 41-75), fut appelé à s'autocontrôler, c'est-à-dire à intérioriser les contraintes liées aux dispositifs organisationnels (Boltanski, Chiapello, 1999/2010, p. 109-118). Ces nouveaux dispositifs furent, au départ, une réponse aux critiques adressées à l'ancienne orga- nisation (hiérarchisée et verticale) de l'entreprise lors du tournant des années 1960-1970 (Boltanski, Chiapello, 1999/2010, p. 69-90). Seulement, les théoriciens du néomanagement, en se débarrassant de la hiérarchie, se sont en même temps débarrassés de l'armature symbolique qui légitimait l'autorité et ses représentants, c'est-à-dire ceux qui avaient la charge et le mandat de représenter dans le groupe la fonction symbolique habituellement dévolue au père. Or c'est à ce moment-là, lors de ce tournant historique qui a vu le capitalisme se transformer, entraînant du même coup ces profonds remaniements dans l'organisation des entreprises et dans leur mode de management, que les phénomènes de harcèlement sont devenus prégnants. Hirigoyen note en effet que le phénomène de harcèlement au travail mute et s'amplifie depuis les années 1970, suivant ainsi comme une ombre les bouleversements organisationnels qui ont touché la sphère de l'entreprise (Hirigoyen, 2001/2013, p. 102-104, 229-264). C'est donc dans l'espace laissé vacant par la figure de l'autorité qu'est venu s'installer le harceleur. Autrement dit, là où s'estompe l'instance du père symbolique (aussi bien dans la famille que dans les institutions), surgissent et se multiplient d'inquiétantes figures paternelles imaginarisant l'Urvater, le père primitif.

Dans chacun de ces cas, les théories enferment le sujet dans la position de victime pour en faire le simple instrument de la jouissance de l'Autre - renforçant ainsi le fantasme sous-jacent. D'où cette seconde remarque : la formule fantasmatique qui s'exprime dans la doctrine de la manipulation mentale n'a pas limité son expansion au champ de la réflexion sur les sectes. Elle s'est infiltrée et diffusée dans de nombreux autres domaines - l'espace domestique, l'univers professionnel -, pour, finalement, contaminer de nombreuses zones de notre culture et, conséquemment, donner aussi corps, tout en le promouvant, au statut de victime. Pourtant, en dépit de sa thèse selon laquelle la victime est strictement passive, M.-F. Hirigoyen ne peut manquer de se laisser surprendre, au détour d'une phrase, par le fantasme masochiste dont elle semble percevoir le contour dans l'étrange comportement des victimes : «Ce qui surprend au premier abord, admet-elle, c'est l'acceptation par les victimes de leur sort » (Hirigoyen, 1998/2001, p. 165-171). Cette résignation suggère, en effet, la part prise des sujets dans leur adhésion au discours qui les instrumentalise. Autrement dit, rien n'indique que ces «victimes » soient réellement à la merci de l'Autre. Certes, certains sujets peuvent être menés à se faire radicalement l'objet de sa 
jouissance - cas des psychotiques mélancoliques ou présentant un état mélancoléiforme qui sont parfois conduits, en radicalisant leur identification à l'objet déchet du symbolique, à se faire, sur un mode littéral, la victime de l'Autre. Pour autant, cette logique mélancolique du sacrifice propitiatoire qui peut pousser les sujets à en passer à l'acte pour restaurer, au prix de leur perte, un monde de toute beauté, demeure rare ${ }^{22}$. En revanche, la fatalité dont les sujets peuvent faire preuve dans l'acceptation de leur sort, masque leur consentement à prendre, selon leurs intérêts propres, position de victime. Cette méconnaissance de leur propre participation, au demeurant active, à s'inscrire sous le statut de victime appelle à s'éclairer.

Pour ce faire, revenons à la question du réel. Tel que Lacan l'entend, le réel n'est pas seulement le référent ultime caché derrière l'écran du fantasme : le réel est à la fois ce qui est caché derrière le voile et le voile lui-même, la tache qui distord la perception de la réalité (Žižek, 2010). Cette tache, ce réel qui fait écran à la perception de la réalité, c'est bien entendu le noyau, énigmatique et fuyant, de notre désir. Or, c'est certainement cette tache dans le tableau qui perturbe le souvenir des ex-adeptes et qui les incite à se dédouaner de leur part de responsabilité, de l'implication de leur désir, dans leur mésaventure sectaire. C'est donc, vraisemblablement, cette tache déformante, soutenue par la doctrine de la manipulation mentale, qui leur permet de faire de l'Autre la cause unique de leur malheur. Ce qui leur évite du même coup d'avoir à assumer les conséquences de leur propre désir, dont ils peuvent ainsi continuer à ne rien vouloir savoir.

C'est ici que la doctrine de la manipulation mentale peut faire le plus de ravages. Elle incite l'ex-adepte à soutenir une position subjective proche du déni ${ }^{23}$, ce qui peut avoir pour effet de paralyser chez lui l'assomption des conséquences de son désir. Le plus grave, bien entendu, est qu'en

22. La logique mélancolique de ce sacrifice propitiatoire, que certains ont épousé sur la scène religieuse (Hamon, 2014, p. 257-270), peut, selon nous, éclairer les attentatssuicides auxquels sont poussés, par résignation ou consentement, des psychotiques engagés, au nom de l'idéal, dans des mouvements sectaires ou extrémistes.

23. La formule qui soutient cette position fantasmatique serait ainsi proche de la formule du déni - « Je sais bien, mais quand même »- promue par Octave Mannoni (Mannoni, 1969, p. 9-33) et, ce faisant, pourrait se déplier ainsi : Je sais bien que l'Autre, qui n'existe pas, est impuissant, mais quand même je préfère continuer à croire qu'il jouit de moi. n'interrogeant rien de cet étrange désir qui a engagé l'adepte sur les voies de son expérience sectaire, l'hypothèse du gourou-manipulateur risque, à l'inverse, de donner de la consistance au fantasme dont on vient d'apercevoir la structure, à savoir le fantasme d'être passivement joui par l'Autre. Autrement dit, non seulement l'hypothèse de la manipulation mentale ne nous enseigne rien des mécanismes libidinaux à l'œuvre dans l'adhésion sectaire, mais, en outre, en nourrissant ce fantasme, elle porte en elle le risque d'engager l'ex-adepte sur les voies de la répétition de cette mésaventure sectaire, qui n'est elle-même que la répétition de la mise en acte de son fantasme.

\section{CONCLUSION : PARANOÏSATION DU LIEN SOCIAL ET RADICALISATION DES CROYANCES}

Le recours à la secte appelle, bien évidemment, à s'interroger au cas par cas afin de cerner la logique subjective qui le sous-tend, la causalité et les fonctions psychiques qu'il remplit, en fonction de la position que le sujet occupe dans la structure clinique. Nous ne pouvons ici entreprendre cette analyse impliquant, outre un questionnement sur la norme et le lien social, de s'interroger sur le sens et les coordonnées des actes d'un sujet. Cette démarche, qui consiste à analyser le recours subjectif à la secte, ouvre en effet à la vérité psychique de l'acte qui le motive, et peut d'ailleurs contribuer, pour qui s'engage dans une cure avec un adepte ou un ex-adepte de secte, à l'intégration par le sujet de sa responsabilité véritable. C'est précisément ce que la théorie de la manipulation mentale évacue car elle répudie, jusqu'à le forclore, le sujet. Plus encore, elle incite insidieusement celui-ci, et lors même qu'elle le dénonce, à se faire objet de jouissance (passivé) et à s'ancrer dans une position de victime. Cette doctrine, outre d'être un symptôme de la dégradation contemporaine de l'instance du père symbolique, œuvre alors dans le sens d'une paranoïsation imaginaire du lien social.

Nous assistons, en effet, à l'installation d'une méfiance généralisée pouvant se résumer au sentiment d'être spolié de son droit à la jouissance par un autre qui, soupçonné de capitaliser celle-ci, prend dès lors les traits d'une figure décadente. La jouissance de l'Autre décadent s'exerçant au détriment du sujet, il n'est pas rare qu'elle soit dénoncée dans sa malignité, au point de le pousser (cet Autre) à théâtraliser, dans la transparence de la scène 
publique, l'aveu de son obscure jouissance ${ }^{24}$. Or on connaît l'étonnante conséquence de cet impératif de transparence : en «mettant tout en lumière, même l'occulte, la société de l'information et de la communication travaille à produire une obscurité plus profonde, à créer de l'inconnu plus lointain, [laissant ainsi] entendre sans l'avoir voulu que la réalité est tout autre que ses apparences, ou que la "vérité est ailleurs" » (Taguieff, 2005, p. 77), là où l'Autre l'a cachée.

Cet idéal de transparence, en entamant ainsi le crédit fait à l'Autre, n'a-t-il pas pour effet l'essor moderne des théories dites du complot ? Ces théories, qui s'inscrivent dans la série des doctrines sur la manipulation mentale - qu'elles élargissent soit à l'échelle du monde ${ }^{25}$, soit à celle du cosmos (Deyo, 2004) -, peuvent alors être envisagées comme des doctrines de la manipulation généralisée : elles prennent acte, à l'échelle des groupes, de deux conséquences de la dégradation de l'instance de l'idéal du moi (support des identifications collectives qui tire sa validité du père symbolique), à savoir la fin des « grands récits » (Lyotard, 1979/2000) et la montée de l'individualisme. Elles visent, pour faire face au délitement du collectif qu'elles envisagent comme le résultat direct des menées d'un Autre malveillant, à construire l'Un d'une communauté homogène. Ces théories se développent donc dans une époque qui, tout en mettant ses espoirs dans la science pour le combler et dans l'idéologie sécuritaire pour le maîtriser, souffre, au fond, du manque de l'Autre de la garantie, battu en brèche par le discours de la science (et ses vérités passagères, soumises au doute méthodique). À cet égard, dans son renversement, la doctrine de la manipulation mentale, d'une part, nous enseigne sur la texture paranoïde du lien social à laquelle elle appartient, tout en délivrant, d'autre part, l'un des motifs principaux de la radicalisation et de l'endoctrinement de nos contemporains. Mettons en effet en hypothèse, et concluons sur cela, que cette doctrine réalise ce qu'elle tente de prévenir.

En avivant toujours plus le sentiment d'être potentiellement victime d'une manipulation, elle

24. Dominique Strauss Kahn a ainsi été tenu de révéler tous les plus obscènes détails de sa vie sexuelle, lors de ses récents procès ultra-médiatiques.

25. David Icke, célèbre théoricien du complot, écrit : « Pour qu'une poignée d'individus puisse diriger et contrôler les masses, quelques structures doivent être en place. Ces structures sont les mêmes qu'il s'agisse de manipuler un particulier, une famille, une tribu, une ville, un pays, un continent ou une planète » (Icke, 2002, p. 27). incite, en effet, à se méfier de l'Autre, de la duplicité du signifiant et, ainsi, à prêter foi en l'idéal d'un savoir absolu construit sur une langue qui serait exacte. La conséquence peut alors être, pour certains sujets, l'adhésion à un discours dans lequel nul principe d'indétermination ne s'insère : là, en ce savoir complet, se situe le noyau d'inertie dialectique qui caractérise la position de ces locuteurs infaillibles que sont les théoriciens conspirationnistes, les gourous et les promoteurs de discours religieux radicalisés. L'hypothèse manipulatoire produit donc deux mouvements paradoxaux : elle donne d'abord corps à un fantasme de structure perverse propice à compléter l'Autre, pour ensuite, une fois cet Autre rendu consistant par la doctrine, tenter de s'en débarrasser en partant en guerre contre ses divers représentants (les innombrables incarnations de la perversité : juifs, francs-maçons, illuminati, reptiliens, grand Satan, etc.). Au premier mouvement - celui où le sujet, soutenu par la logique du fantasme masochiste qui sous-tend la notion de manipulation mentale, se met en position de victime -, succède ainsi un second mouvement, paranoïaque, de localisation de la jouissance dans l'Autre, susceptible d'inciter nombre de nos contemporains à rejoindre l'un des discours « radicaux » sans faille - puisque fondés sur le rejet du point d'inconsistance autorisant le doute - qui pullulent depuis plusieurs dizaines d'années. Autrement dit, non seulement les doctrines de la manipulation mentale risquent d'engager les sujets sur la voie de la répétition de leur expérience sectaire, mais elle pourrait même en inciter certains à se radicaliser en adhérant à des doctrines paranoïaques, au nom d'une aspiration à un Un ${ }^{26}$ débarrassé de l'Autre, le jouisseur pervers ${ }^{27}$.

26. La communauté imaginaire des alter-ego : aussi bien la «Oumma » des djihadistes de Daech que la communauté intégralement « clarifiée » des scientologues.

27. L'Occident dévoyé pour les djihadistes, la psychiatrie pour les scientologues. 


\section{RÉFÉRENCES}

Abelhauser (Alain).- L'imposture, partenairesymptôme de la science, Cliniques méditerranéennes, I, $81,2010$.

Abgrall (Jean-Marie).- La mécanique des sectes [1996], Paris, Payot, 2000.

Anthony (Dick).- Pseudoscience and minority religions: An evaluation of the brainwashing theories of Jean-Marie Abgrall, Social Justice Research, XII, 4, 1999, p. 421-456.

Anthony (Dick), Introvigne (Massimo).-Le lavage de cerveau, mythe ou réalité ?, Paris, L'Harmattan, 2006.

ArIÈs (Paul).- La scientologie : laboratoire du futur?, Lyon, Golias, 1998.

BERnays (Edward).-Propaganda: comment manipuler en démocratie [1928], Paris, La Découverte, 2007.

Boltanski (Luc), Chiapello (Ève). - Le nouvel esprit du capitalisme [1999], Paris, Gallimard, 2010.

Bouderlique (Max).- Sectes : les manipulations mentales, Lyon, Chronique Sociale, 1990.

BouderliQue (Max).- Comprendre l'action des sectes, Lyon, Chronique Sociale, 1995.

Boudon (Raymond).- Effets pervers et ordre social, Paris, Presses universitaires de France, 1993.

Bourin (Guillaume Xavier).- Contribution à l'étude du délit de manipulation mentale préjudiciable, thèse de doctorat, Université Montpellier 1, 2003.

Bouzar (Dounia).- Comment sortir de l'emprise " djihadiste »?, Ivry-sur-Seine, Éditions de l'Atelier, 2015.

BRoDSKy (Carroll).- The Harrassed Worker, Lexington, Health and Company, 1976.

Castanet (Didier).- Du désir à la cause et au fantasme, Trèfle, 4-5, 2002.

Champion (Françoise), Cohen (Martine).- Sectes et démocraties, Paris, Seuil, 1999.

Chouvier (Bernard), Morhain (Yves).- Position sectaire, croyance et emprise groupale, Revue de psychothérapie psychanalytique de groupe, II, 49, p. 25-38, 2007.

Corraze (Jacques).- De l'hystérie aux pathomimies, Paris, Dunod, 1976.

Dericquebourg (Régis).- La mécanique des sectes : étude scientifique ou discours militant ? [le 7 juillet 2015, http://www.regis-dericquebourg.com/2011/10/10/ la-mecanique-des-sectes-etude-scientifique-ou-discoursmilitant/].

Deyo (Stan).- La conspiration cosmique, Québec, Louise Courteau, 2004.

DUPRÉ (Ernest).- La mythomanie, étude psychologique et médico-légale du mensonge et de la fabulation morbides, Paris, J. Gainche, 1905.

DiET (Emmanuel).- De la perversité à la perversion sexuelle dans les groupes sectaires, Revue de psychothérapie psychanalytique de groupe, II, 43, 2004, p. 79-91.

ESQUERRE (Arnaud).- Du psychopouvoir : une sociologie de la manipulation mentale dans le cadre de la lutte contre les « sectes » en France dans les années 2000, thèse de doctorat, EHESS, 2008.

Fournier (Anne), Monroy (Michel).- La dérive sectaire, Paris, Presses universitaires de France, 1999.

Fournier (Anne).- La dérive sectaire, Le journal des psychologues, 174, 2000, p. 23-27.

Fournier (Anne).- Postface, dans Maes (J.-C.), Emprise et manipulation, Bruxelles, De Boeck supérieur, 2010, p. 255-257.

Freud (Sigmund).- Lettres à Wilhelm Fliess, 18871904, Édition complète, Paris, Presses universitaires de France, 2006.

Freud (Sigmund), Breuer (Joseph).- Études sur l'hystérie [1895], Paris, Presses universitaires de France, 2005.

Hamon (Romuald).- Le Padre Pio, une mélancolie magnifiée par la religion catholique, Cliniques méditerranéennes, 89, 2014, p. 257-270.

Hervieu-LÉGer (Danièle).- La religion en miettes ou la question des sectes, Paris, Calmann-Levy, 2001.

Hervieu-Léger (Danièle).- Le partage du croire religieux dans des sociétés d'individus, L'Année sociologique, 60, I, 2010, p. 41-62.

Hirigoyen (Marie-France).- Le harcèlement moral [1998], Paris, La découverte et Syros, 2001.

Hirigoyen (Marie-France).- Le harcèlement moral dans la vie professionnelle [2001], Paris, La découverte et Syros, 2013.

Hubbard (L. Ron).- La Dianétique : la puissance de la pensée sur le corps [1950], Copenhague, New Era Publication International ApS, 2003. 
ICKE (David).- Les enfants de la matrice, 1, Québec, Courteau Louise, 2002.

LACAN (Jacques).- Les complexes familiaux dans la formation de l'individu [1938], dans Autres écrits, Seuil, Paris, 2001.

LACAN (Jacques).- D'une question préliminaire à tout traitement possible de la psychose [1959], dans Écrits, Paris, Seuil, 1966, p. 531-583.

LaCAN (Jacques).- Subversion du sujet et dialectique du désir dans l'inconscient freudien [1960], dans Écrits, Paris, Seuil, 1966, p. 793-827.

LACAN (Jacques).- Le séminaire, Livre XI, Les quatre concepts fondamentaux de la psychanalyse, Paris, Seuil, 1973.

LaCAN (Jacques).- Le séminaire, Livre XX, Encore, Paris, Seuil, 1975.

LACAN (Jacques).- Le séminaire, Livre XVII, L'envers de la psychanalyse, Paris, Seuil, 1981.

Lenolr (Frédéric), Luca (Nathalie).- Sectes : mensonges et idéaux, Paris, Bayard, 1998.

LEYMANN (Heinz).- La persécution au travail [1993], Paris, Seuil, 2002.

LoI $n^{\circ}$ 2001-504 du 12 juin 2001 (dite loi AboutPicard) tendant à renforcer la prévention et la répression des mouvements sectaires portant atteinte aux droits de l'homme et aux libertés fondamentales [le 13.11.2015, http://www.legifrance.gouv.fr/affichTexte.do?cidTexte=J ORFTEXT000000589924\&dateTexte=].

LYOTARD (Jean-François).- La condition postmoderne [1979], Paris, Les éditions de Minuit, 2000.

Maes (Jean-Claude).- La « chose » sectaire, Psychothérapies, III, 22, 2002, p. 175-188.

MAEs (Jean-Claude).- Le lien sectaire : des relations fondées sur la rupture, Thérapie familiale, II, 27, 2006, p. 133-159.

Maes (Jean-Claude).- La collusion sectaire, Dialogues, 145,1999 , p. 27-50.

MAEs (Jean-Claude).- Emprise et manipulation, Bruxelles, De Boeck supérieur, 2010.

Mannoni (Octave).- Je sais bien, mais quand même..., dans Clefs pour l'imaginaire : ou l'Autre scène, Paris, Seuil, 1969, p. 9-33.

Marx (Karl), Engels (Friedrich).- Manifeste du Parti communiste [1848], Paris, Bordas, 1989.

Marx (Karl).- Philosophie [1935-1867], Paris, Gallimard, 1994.

Marzano (Michela).- Extension du domaine de la manipulation. De l'entreprise à la vie privée [2008], Paris, Fayard, 2010.

Milgram (Stanley).- Soumission à l'autorité, Paris, Calmann-Lévy, 1974.

Miscavige Hill (Jenna), Pulitzer (Lisa).- Rescapée de la scientologie, Paris, Kero, 2013.

Miviludes.- Les dérives sectaires, Année 2003 [le 6 juillet 2015, http://www.derives-sectes.gouv. $\mathrm{fr} / \mathrm{sites} /$ default/files/publications/francais/Rapport_ MIVILUDES_2003.pdf].

Miviludes.- Le risque sectaire, Année 2004 [le 6 juillet 2015, http://www.derives-sectes.gouv.fr/ sites/default/files/publications/francais/RAPPORT_ MIVILUDES_2004.pdf].

Miviludes.- Rapport au Premier Ministre, Année 2006 [le 6 juillet 2015, http://www.derives-sectes.gouv. $\mathrm{fr} / \mathrm{sites} / \mathrm{default} /$ files/publications/francais/rapport_ miviludes_2006.pdf].

Nathan (Tobie), Swertvaegher (Jean-Luc).- Sortir d'une secte, Paris, Seuil, 2003.

Norel (Alice).-Sectes religieuses, croyances et relation d'emprise, thèse de doctorat, Université Paris 5, 2006.

PÉrÉrA (Eric).- La production du body-builder : ascèse, emprise et lien sectaire, thèse de doctorat, Université Montpellier 3, 2010.

RACAMIER (Paul-Claude).- La perversion narcissique [2012], Paris, Payot, 2013.

SaUvayre (Romy).- Le processus d'abandon des croyances défiant le sens commun, thèse de doctorat, Université de Strasbourg, 17 novembre 2010.

SINGER (Margaret).- Cults in our Midst: The continuing Fight against Their Hidden Menace, San Francisco, Jossey-Bass, 2003.

Smith (Adam).- La richesse des nations, II [1776], Paris, Flammarion, 1999.

TAguiefF (Pierre-André).- La foire aux illuminés, Paris, Mille et une nuits, 2005.

VAsquez (Mona).- Et Satan créa la Secte. Mémoires d'une rescapée, Toulouse, Messages, 2004.

Verlinde (Joseph-Marie).- Les racines cachées du Nouvel Âge, Nouvelle revue théologique, III, 125, 2003, p. 353-373.

ŽIžEK (Slavoj).- Ils ne savent pas ce qu'ils font : le sinthome idéologique, Paris, Point Hors Ligne, 1990.

ŽIžEK (Slavoj).- Jacques Lacan à Hollywood, et ailleurs [1992], Paris, Éditions Jacqueline Chambon, 2010. 